REVIEW

\title{
Lupus nephritis: An approach to diagnosis and treatment in South Africa
}

\author{
I G Okpechi, ${ }^{1} \mathrm{MBBS}$, FWACP, PhD, Cert Nephrology (SA); A Gcelu, ${ }^{2} \mathrm{MB}$ ChB, FCP (SA), Cert Rheumatology (SA); \\ O I Ameh, ${ }^{1}$ MBBS, FMCP Neph (Nigeria), Cert Nephrology (SA) \\ ${ }^{1}$ Division of Nephrology and Hypertension, Department of Medicine, Faculty of Health Sciences, Groote Schuur Hospital and
University of Cape Town, South Africa
${ }^{2}$ Division of Rheumatology, Department of Medicine, Faculty of Health Sciences, Groote Schuur Hospital and University of Cape Town, South Africa
}

Corresponding author: I G Okpechi (ikechi.okpechi@uct.ac.za)

Lupus nephritis (LN) is a significant cause of morbidity and mortality in patients with systemic lupus erythematosus. Delayed recognition and diagnosis of LN may be a common cause of chronic kidney disease among South Africans. Renal biopsy is the gold standard of diagnosing LN; however, this service is not available in many centres and the use of urinalysis, urine microscopic examination and other serological tests can be useful in identifying patients with proliferative LN. Proliferative types of LN (class III, class IV and mixed class V) comprise the larger proportion of patients with this condition. Patients receiving immunosuppressive therapy need to be monitored closely for side-effects and drug-related toxicities. LN patients with end-stage renal disease (class VI) need to be prepared for renal replacement therapy (dialysis and renal transplantation). In all patients, treatment should include adjunctive therapies such as renin angiotensin aldosterone system blockade, bone protection (with calcium supplements and vitamin D), blood pressure control and chloroquine - all of which help to retard the progression of kidney disease.

S Afr Med J 2015;105(12):XXX. DOI:10.7196/SAMJ.2015.v105i12.10224

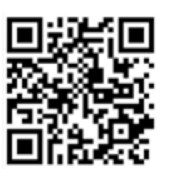

Definition and epidemiology of lupus nephritis

Systemic lupus erythematosus (SLE) is a chronic multi-systemic autoimmune disorder with a predilection for young females. Kidney disease in SLE (also known as lupus nephritis (LN)) is a common manifestation of SLE and constitutes an important prognostic factor for such patients. Up to $50 \%$ of SLE patients have abnormalities of renal function or urine (proteinuria, haematuria or cellular casts) early in the course of the disease, whereas approximately $80 \%$ may later develop overt abnormalities of renal function. ${ }^{[1]}$ According to the 2012 Systemic Lupus International Collaborating Clinics Classification (SLICC) criteria for the diagnosis of SLE, kidney disease is present when a patient with SLE presents with persistent proteinuria $(>0.5 \mathrm{~g} / 24 \mathrm{~h})$ or cellular red cell casts. ${ }^{[2]} \mathrm{LN}$ is a major determinant of morbidity and mortality in SLE patients. A South African (SA) study has shown that over half of 226 SLE patients from a lupus clinic had either died or been lost to follow-up at 55 months, and LN was the only significant factor associated with mortality on multivariate analysis, with a 5 -year survival rate of $60 \% \cdot{ }^{[3]}$

\section{Classification}

The classification of LN is based on histological features, using the International Society of Nephrology (ISN) and Renal Pathology Society (RPS) criteria developed in 2003 (Table 1). ${ }^{[4]}$ Although the classification is mainly glomerulocentric, it includes features of tubulo-interstitial disease, from which features of chronicity can be determined. The relationship between the histological class of LN and clinical course of the disease is well recognised. Patients with class II and class V (pure membranous LN) disease usually have a slow decline in renal function over long periods of observation. In contrast, patients with class III and class IV (or those with mixed class $\mathrm{V}$ ) disease mostly have a more aggressive course of disease. Various studies have shown that the proliferative forms of LN (i.e. class III, class IV and mixed class V) occur more frequently than the other histological morphologies. In Cape Town, of 251 patients with biopsy-proven LN, 63\% had proliferative LN. ${ }^{[5]}$

\section{Diagnosis}

One of the major challenges with regard to SLE is its early recognition and diagnosis. Many physicians assume that SLE is a rare condition in Africa - it is therefore seldom considered as a differential diagnosis, except when patients present with classic features, such as malar rash and swollen painful joints of the hands and feet. Hence, many patients tend to remain ill for prolonged periods before the diagnosis is made. In some instances, chronic damage to organs including the kidneys would have occurred.

\section{Role of urinalysis and urine microscopy}

LN is unlikely to present alone - it often manifests with other extrarenal features such as joint pain, malar rash, oral ulcers and photosensitivity. The

Table 1. Abbreviated International Society of Nephrology/Renal Pathology Society classification of lupus nephritis (2003) ${ }^{[4]}$

\begin{tabular}{ll}
\hline Class I & Minimal mesangial lupus nephritis \\
Class II & Mesangial proliferative lupus nephritis \\
Class III & Focal lupus nephritis (<50\% involvement) \\
& $\begin{array}{l}\text { Diffuse segmental or diffuse global lupus nephritis } \\
\text { ( } \geq 50 \% \text { involvement })\end{array}$ \\
Class IV & Membranous lupus nephritis ${ }^{*}$ \\
Class V & Advanced sclerosing lupus nephritis \\
Class VI & ${ }^{*}$ Can be pure membranous lupus nephritis or mixed class V if combined with class III or class IV.
\end{tabular}


patient with LN is likely to present with nephritic syndrome (oliguria, minimal proteinuria, haematuria, hypertension and azotaemia) or with features of nephrotic syndrome (anasarca, heavy proteinuria and hypoalbuminaemia). Urinalysis (dipstick and microscopic examination) presents the best opportunity for early identification of LN as a dipstick is likely to show the presence of blood and protein in the urine and urine microscopy enables identification of various urinary casts (red cells, granular, hyaline). LN must be strongly suspected in any SLE patient with high titres of double-stranded DNA (dsDNA) and a positive dipstick for blood. Urinalysis features have been shown to be correlated with the presence of proliferative class LN. ${ }^{[5]}$ Therefore, every SLE patient should have a urinalysis performed at every clinic visit.

\section{Role of lupus auto-antibodies (antinuclear antibody and dsDNA) and complements $(\mathrm{C} 3 / \mathrm{C} 4)$}

Auto-antibodies in SLE and complements $(\mathrm{C} 3 / \mathrm{C} 4)$ are known to be elevated or lowered, respectively, in patients with increased disease activity, especially in those with proliferative LN (class III, class IV and mixed class V). One study found proliferative LN to be significantly correlated with haematuria on dipstick $(p<0.0001)$, proteinuria on dipstick $(p=0.042)$, low complement C3 ( $p<0.0001)$, low complement $\mathrm{C} 4(p=0.009)$ and positive dsDNA $(p=0.039) .^{[5]}$

\section{Role of renal biopsy}

The definitive diagnosis of $\mathrm{LN}$ requires a kidney biopsy. Renal histology also enables classification of $\mathrm{LN}$ and assists in the prognosis. All guidelines recommend a renal biopsy when there is a suspicion of renal involvement, as clinical and laboratory parameters, although useful, cannot accurately predict the histological class. The indication for a renal biopsy in SLE patients includes persistent decline in renal function, proteinuria $(\geq 1.0 \mathrm{~g} / 24 \mathrm{~h})$ or proteinuria $(\geq 0.5 \mathrm{~g} / 24 \mathrm{~h})$ if associated with haematuria (5 red blood cells (RBCs)/high-power field) and active urinary sediment (granular casts, white blood cell (WBC) casts, RBC casts). It is recommended that the biopsy be examined by light microscopy, immunofluorescence (or immunohistochemistry) and where possible by electron microscopy. ${ }^{[6]}$ Quantification of activity and chronicity indices and description of vascular and interstitial lesions are also recommended. A repeat renal biopsy is indicated if there is evidence of worsening of the disease or disease refractory to treatment, evidence of relapse (to show transformation or progression in histological class or change in activity and chronicity cores) and to demonstrate other pathologies. Fig. 1 shows the renal histology in a patient with class IV LN.

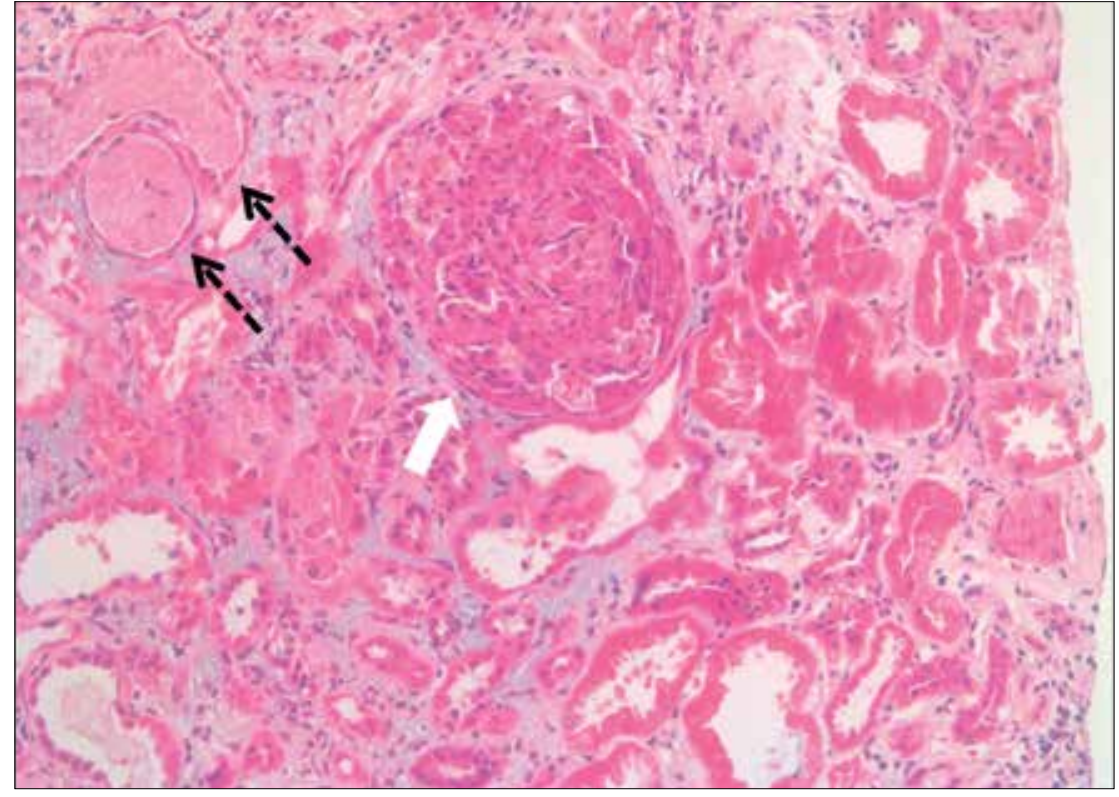

Fig. 1. Renal histology of a patient with class IV lupus nephritis (white arrow shows a glomerulus with cellular crescent; black broken arrows show adjacent tubules with red cell casts).

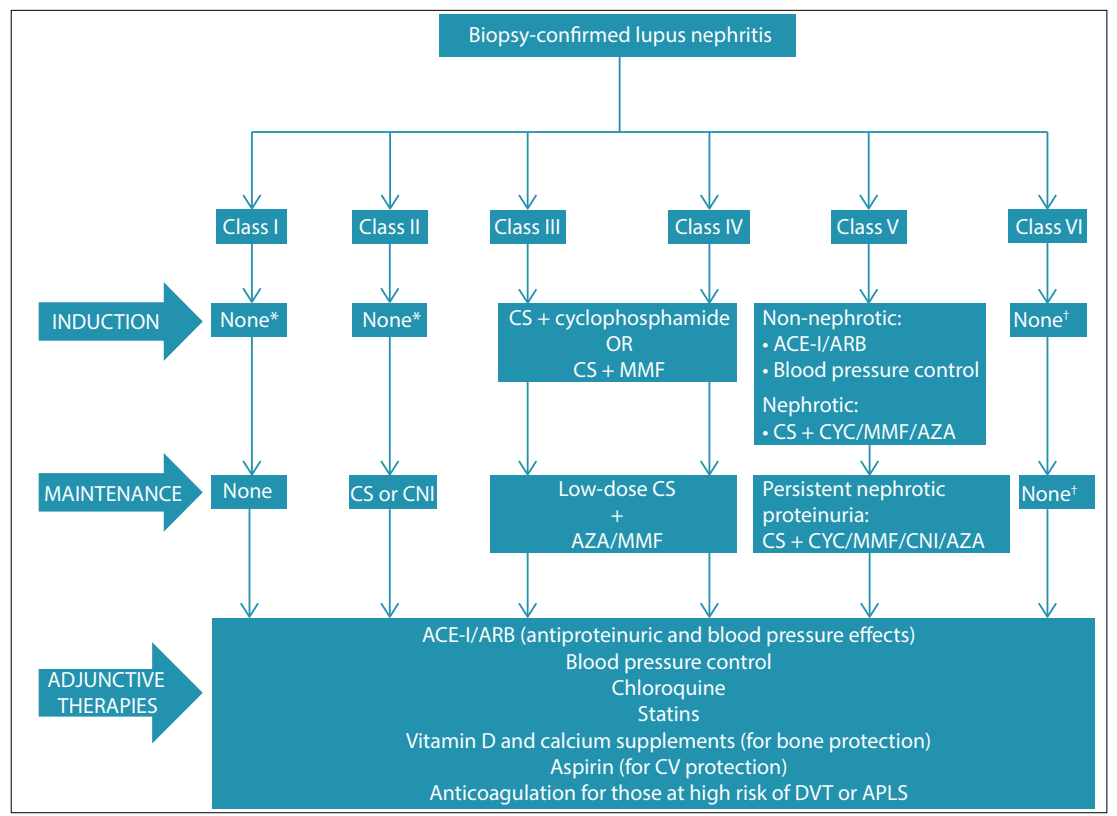

Fig. 2. Treatment approach for patients with lupus nephritis (APLS = antiphospholipid syndrome; $A Z A=$ azathioprine; $A C E-I=$ angiotensin converting enzyme inhibitor; $A R B=$ angiotensin receptor blocker; $C N I=$ calcineurin inhibitor; $C S=$ corticosteroid; $C Y C=$ cyclophosphamide; $D V T=$ deep-vein thrombosis; $M M F=$ mycophenolate mofetil; $C V=$ cardiovascular).

* Immunosuppression to be dictated by extrarenal manifestations.

Patients should be prepared for renal replacement therapy (dialysis/ transplantation).

\section{Treatment}

The treatment of LN is dictated by the class of the disease and degree of activity and chronicity indices. All patients should receive adjuvant therapies as indicated and if tolerated. There should be a riskbenefit evaluation when deciding whether to use immunosuppression in patients with increased chronicity indices (i.e. glomerular sclerosis with tubulo-interstitial fibrosis) because of the increased risk of side-effects of treatment. The approach to treatment often involves two phases for patients with proliferative LN (class III, class IV and mixed class $\mathrm{V}$ ).

\section{Induction therapy}

Fig. 2 summarises the common approaches used for induction for the different classes of LN. ${ }^{[7,8]}$ Induction therapy is not used for 
patients with class I, II, V (pure class $\mathrm{V}$ with sub-nephrotic range proteinuria) and VI LN. The approach to induction involves the use of 3 consecutive pulses of intravenous methylprednisolone (500 - $750 \mathrm{mg}$ daily) together with another immunosuppressive: cyclophosphamide (CYC) (daily oral or monthly intravenous pulse therapy) or mycophenolate mofetil (MMF). The patient should continue on oral prednisone $(1 \mathrm{mg} / \mathrm{kg} /$ day $)$ after completing the pulse treatment with methylprednisolone. CYC is often the agent of choice for many clinicians owing to easy accessibility and cost; however, the use of MMF is increasing. Various studies have assessed the efficacy and safety of CYC with MMF or placebo for induction therapy in patients with LN (reviewed in depth by $\mathrm{Chan}^{[9]}$ ). The recommended duration of induction therapy is 6 months; during this time, the dose of oral corticosteroid should be weaned.

\section{Maintenance therapy}

Treatment administered during the induction phase of therapy is de-escalated in the maintenance phase. The goal of the latter phase is to maintain the response (remission) gained during the induction phase and therefore to retard progression of chronic kidney disease. Immunosuppressive agents commonly used include MMF, azathioprine (AZA), and corticosteroids. Calcineurin inhibitors may be used in special circumstances, such as in cases of intolerance to MMF or AZA or in patients with persistent heavy proteinuria (Fig. 2). There is currently no consensus on the duration of maintenance therapy. Nonetheless, the decision to withdraw maintenance immunosuppression should be guided by sustained complete clinical response over a period of at least 2 years. Withdrawal should be done gradually, starting with glucocorticoids before withdrawing immunosuppressive agents. ${ }^{[8]}$ In debilitating or life-threatening complications of immunosuppression, therapy should be withdrawn

\section{Adjunctive therapies}

Adjunctive therapies are usually started during the induction phase of treatment and although some need to be discontinued after completing this therapy, others will need to be continued during the maintenance phase. Commonly recommended adjunctive therapies in LN include:

- Renin angiotensin aldosterone system (RAAS) inhibition for proteinuria and blood pressure treatment (target $<130 / 80 \mathrm{mmHg}$ ).

- Bone protection with calcium and vitamin D supplements.

- Chloroquine for all patients (unless contraindicated, e.g. visual disturbance).

- Treatment of hyperlipidaemia with statins (target low-density lipoprotein $<2.6 \mathrm{mmol} / \mathrm{L}$ ).
- Low-dose acetylsalicylic acid in patients with antiphospholipid syndrome.

- Anticoagulant to be considered in patients with nephrotic syndrome and albumin $<20 \mathrm{~g} / \mathrm{L}$

- Avoid vaccination with live or attenuated viruses during immune suppression.

- Tuberculosis prophylaxis with isoniazid (for those in highly endemic TB regions).

\section{Treatment of refractory $L N$}

Fewer than $50 \%$ of patients are able to achieve complete remission during the 6 months of induction therapy. It may take up to 2 years to reach remission in many patients. Switching to an alternative agent is recommended for patients who fail to improve within 3 - 4 months, or do not achieve a partial response after 6 - 12 months or a complete response after 2 years of treatment. Treatment options include switching from MMF to CYC or from CYC to MMF; rituximab may be given as add-on treatment or monotherapy. Other options include the use of calcineurin inhibitors, intravenous immunoglobulin and plasma exchange for patients with rapidly progressive glomerulonephritis.

\section{Treatment of class VI LN}

Immunosuppression for class VI patients must be dictated by extrarenal manifestations of SLE. These patients should be prepared for renal replacement therapy (haemodialysis, peritoneal dialysis or transplantation). Treatment and prevention of cardiovascular risk factors (e.g. blood pressure control, statins for dyslipidaemia) should be continued.

\section{Monitoring for drug toxicities and disease activity}

It is recommended that body weight, blood pressure, serum creatinine, urinalysis (for proteinuria and urinary sediment), C3/C4, anti-dsDNA, serum albumin and full blood count be performed at each clinic visit. Patients with active nephritis should be seen monthly or more frequently, while those with no active disease should be seen twice yearly or quarterly. This ensures monitoring of disease activity. Useful approaches to monitoring for drug toxicities/side-effects are summarised in Table 2.

\section{Treatment options for pregnant $\mathrm{LN}$ patients}

Pregnant LN patients often present a challenge to the clinician, as pregnancy can be a trigger for increased disease activity. Some guidelines therefore recommend that pregnancy should not be planned until remission has been achieved and maintained for at least 6 months. ${ }^{[7,8]}$ Pregnant patients with LN have an increased risk of pre-eclampsia, preterm delivery and fetal loss. They should therefore be managed by a multidisciplinary team

Table 2. Adverse effects of drugs and approach to monitoring in patients with lupus nephritis

\begin{tabular}{|c|c|c|}
\hline Medication & Adverse effect & Monitoring approach \\
\hline Glucocorticoids & $\begin{array}{l}\text { Glucose intolerance/diabetes mellitus, osteoporosis, hypertension, } \\
\text { gastritis, cataracts, avascular necrosis of femur, cushingoid appearance }\end{array}$ & $\begin{array}{l}\text { Blood glucose measurement, bone mineral density } \\
\text { assessment, blood pressure monitoring, weight monitoring }\end{array}$ \\
\hline Cyclophosphamide & $\begin{array}{l}\text { Leukopenia, menstrual disorders, gonadal failure, } \\
\text { haemorrhagic cystitis }\end{array}$ & Full blood count, urinalysis \\
\hline $\begin{array}{l}\text { Mycophenolate } \\
\text { mofetil/ } \\
\text { mycophenolic acid }\end{array}$ & Leukopenia, hypercholesterolaemia & $\begin{array}{l}\text { Full blood count, serum cholesterol, gastrointestinal } \\
\text { disturbances and neurotoxicity assessment }\end{array}$ \\
\hline Azathioprine & Leukopenia, hepatotoxicity, pancreatitis & $\begin{array}{l}\text { Full blood count, liver function tests, dose adjustment } \\
\text { for concomitant allopurinol use }\end{array}$ \\
\hline Chloroquine & Retinopathy & Annual ophthalmology examination \\
\hline
\end{tabular}


Table 3. Use of immunosuppressive and adjunctive agents in pregnancy

\begin{tabular}{lll}
\hline Drug & $\begin{array}{l}\text { May the drug be } \\
\text { used in pregnancy? }\end{array}$ & Side-effects \\
\hline Glucocorticoids & Yes & $\begin{array}{l}\text { High risk of gestational diabetes and } \\
\text { PROM }\end{array}$ \\
$\begin{array}{lll}\text { Cytotoxic agents } & \\
\text { - Cyclophosphamide } & \text { No } & \text { Increased fetal loss and abnormalities } \\
\text { - Azathioprine } & \text { Yes } & \text { - } \\
\text { - Mycophenolate mofetil } & \text { No } & \text { May cause congenital abnormalities } \\
\text { - Chloroquine } & \text { Yes } & \text { - } \\
\text { Calcineurin inhibitors } & & \\
\text { - Cyclosporin } & \text { Yes } & \text { Increased risk of obstetric cholestasis } \\
\text { - Tacrolimus } & \text { Yes } & \text { Increased risk of gestational diabetes } \\
\text { - Rituximab } & \text { No } & \text { Lack of data } \\
\text { RAAS blockade } & \text { No } & \begin{array}{l}\text { May cause congenital defects in the renal, } \\
\text { pulmonary, cardiac, skeletal and } \\
\text { nervous systems }\end{array} \\
\text { PROM = premature rupture of membranes; RAAS = renin angiotensin aldosterone system. Adapted from Day CJ, et al. }{ }^{[10]}\end{array}$
\end{tabular}

of clinicians (obstetricians, rheumatologists and nephrologists). Angiotensin converting enzyme inhibitors, angiotensin receptor blockers, MMF and $\mathrm{CYC}$ are prohibited during pregnancy (Table 3). ${ }^{[10]}$ Prednisone, AZA and calcineurin inhibitors may be continued during pregnancy. Labetalol, methyldopa and nifedipine may be used for control of blood pressure, and lowdose acetylsalicylic acid should be considered to reduce the risk of pre-eclampsia.

\section{Definitions of response to treatment and flares in LN}

There is consensus for defining a complete response as inactive urinary sediment, a decrease in proteinuria to $\leq 0.2 \mathrm{~g} /$ day and normal or stable renal function (within $10 \%$ of normal glomerular filtration rate (GFR) if previously abnormal). ${ }^{[1]}$ Partial response is taken as improvement of renal function, marked by inactive sediment, proteinuria $\leq 0.5 \mathrm{~g} /$ day, with normal GFR or stable renal function. A flare is defined as an increase in disease activity requiring more intensive therapy. It is indicated by an increase in proteinuria or serum creatinine, abnormal urinary sediment or a reduction in creatinine clearance due to active disease. There are two main types of flare:

- Nephrotic flare - proteinuria $>2 \mathrm{~g} /$ day.
- Nephritic flare typified by an increasing anti-dsDNA, low C3/C4, active urine sediment, and worsening serum creatinine.

LN flare surveillance during visits includes urinanalysis (dipstick and microscopy) for proteinuria and cellular casts such as RBC casts, WBC casts, and serum creatinine determination. Significant proteinuria on dipstick analysis should be followed by a quantitative protein analysis.

\section{References}

1. Cameron JS. Lupus nephritis. J Am Soc Nephrol 1999;10:413424.

2. Petri M, Orbai AM, Alarcón GS, et al. Derivation and validation of the Systemic Lupus International Collaborating Clinics classification criteria for systemic lupus erythematosus. Arthritis Rheum 2012;64:2677-2686. [http://dx.doi.org/10.1002/art.34473]

3. Wadee S, Tikly M, Hopley M. Causes and predictors of death in South Africans with systemic lupus erythematosus. death in South Africans with systemic lupus erythematosus.
Rheumatology 2007;46:1487-1491. [http://dx.doi.org/10.1093/ Rheumatology 2007;46:
rheumatology/kem180]

rheumatology/kem 180]
. Weening JJ, D'Agati VD, Schwartz MM, et al. The classification of glomerulonephritis in systemic lupus erythematosus revisited. J Am Soc Nephrol 2004;15:241-250. [http://dx.doi.org/10.1097/01. ASN.0000108969.21691.5D]

5. Okpechi IG, Swanepoel CR, Tiffin N, et al. Clinicopathological insights into lupus nephritis in South Africans: A study of 251 patients. Lupus 2012;21:1017-1024. [http://dx.doi. org/10.1177/0961203312441981] 6. Wilhelmus S, Bajema IM, Bertsias GK, et al. Lupus nephritis management guidelines compared. Nephrol Dial Transplant 2015, 28

7.

Kidney Disease: Improving Global Outcomes (KDIGO) Glomerulonephritis Work Group. KDIGO Clinical Practice Guideline for Glomerulonephritis. Kidney Int 2012;2:139-274

8. Bertsias GK, Tektonidou M, Amoura Z, et al. Joint European League Against Rheumatism and European Renal AssociationEuropean Dialysis and Transplant Association (EULAR/ERAEDTA) recommendations for the management of adult and paediatric lupus nephritis. Ann Rheum Dis 2012;71:1771-1782. [http://dx.doi.org/10.1136/annrheumdis-2012-201940]

9. Chan TM. Treatment of severe lupus nephritis: The new horizon. Nat Rev Nephrol 2015;11:46-61. [http://dx.doi.org/10.1038/ nrneph.2014.215]

10. Day CJ, Lipkin GW, Savage CO. Lupus nephritis and pregnancy in the 21st century. Nephrol Dial Transplant 2009;24:344-347. [http://dx.doi.org/10.1093/ndt/gfn651]

11. Gordon C, Jayne D, Pusey C, et al. European consensus statement on the terminology used in the management of lupus glomerulonephritis. Lupus 2009;18:257-263. [http://dx.doi. org/10.1177/0961203308100481] 